\title{
Hepatic sonic hedgehog protein expression measured by computer assisted morphometry significantly correlates with features of non-alcoholic steatohepatitis
}

Michael Estep ${ }^{1,2}$, Rohini Mehta ${ }^{1,2}$, Gary Bratthauer ${ }^{2}$, Lakshmi Alaparthi ${ }^{1,2}$, Fanny Monge ${ }^{1,2}$, Simon Ali ${ }^{1}$, Dinan Abdelatif2 ${ }^{2}$, Zahra Younoszai ${ }^{2}$, Maria Stepanova ${ }^{3}$, Zachary D. Goodman ${ }^{1,2}$ and Zobair M. Younossi ${ }^{1,2^{*}}$

\begin{abstract}
Background: Hepatic expression of Sonic Hedgehog (SHH) is associated with Non-alcoholic fatty liver disease (NAFLD) and development of Non-alcoholic steatohepatitis (NASH). Hepatic SHH detection increases with the diagnosis of NASH. This pilot study was designed to confirm that staining for $\mathrm{SHH}$ is useful in NASH diagnosis and determine whether quantification of staining by computer assisted morphometry (CAM) can be used to assess severity of ballooning degeneration.

Methods: SHH was detected by immunohistochemistry $(\mathrm{IHC})$ on paraffin-embedded liver sections in subjects $(N=69)$ with biopsy proven NAFLD and no liver disease (control). Serum samples were also available for these subjects. Post-staining, a digitized image of the section was acquired and an area quantification algorithm was used to quantify the degree of $\mathrm{SHH}$ expression. Additionally, circulating M30, M65, and SHH were measured by ELISA.

Results: Notably, hepatic SHH expression correlated with histologic ballooning degeneration (rho $=0.62, p<0.0001$ ), steatosis grade ( $r h o=0.554, P<0.001$ ), Mallory-Denk bodies $(r h o=0.54, P<0.001)$, pericellular fibrosis ( $r h o=0.527$, $P<0.001$ ), and lymphocytic infiltration (rho $=0.435, P<0.0002$ ). Additionally, hepatic SHH expression correlated with circulating M65 (rho $=0.588, p<0.0001)$, and circulating M30 (rho $=0.375, p=0.001)$, as well as AST and ALT (rho $=0.43$, $p=0.0004$, and rho $=0.27, p=0.03$, respectively). Further, serum M30 was almost twice as high in NASH patients compared to non-NASH (539.1 $\pm 290.8 \mathrm{U} / \mathrm{L}$ vs. $287.6 \pm 190.5 \mathrm{U} / \mathrm{L} ; p=0.0002)$, while M65 was almost three times higher in NASH patients compared to non-NASH $(441.2 \pm 464.2 \mathrm{U} / \mathrm{L}$ vs. $162.8 \pm 353.1 \mathrm{U} / \mathrm{L}, P=0.0006)$. Logistic modeling indicates hepatic $\mathrm{SHH}$ expression and presence of type 2 diabetes as independent predictors of advanced fibrosis (defined as portal and pericellular fibrosis $>2: \mathrm{OR}=1.986, p=0.01$, and $\mathrm{OR}=3.280, p=0.03$, respectively).

Conclusion: Thus, our findings show quantitation of SHH expression by CAM can provide a tool for quantifying changes in hepatocyte injury and assist in unambiguous staging/grading of NASH. Our study showed minimal interobserver variability using CAM based quantification. Once validated, CAM assessment of hepatic SHH could benefit clinical trials or long term outcomes studies of NASH subjects.
\end{abstract}

Keywords: NAFLD, Ballooning degeneration, NASH, Hedgehog

\footnotetext{
* Correspondence: zobair.younossi@inova.org

${ }^{1}$ Center for Liver Diseases, Department of Medicine, Inova Fairfax Hospital, Virginia, USA

${ }^{2}$ Betty and Guy Beatty Center for Integrated Research, Claude Moore Health Education and Research Building, Inova Health System, 3300 Gallows Road, Falls Church, VA 22042, USA

Full list of author information is available at the end of the article
}

(c) The Author(s). 2019 Open Access This article is distributed under the terms of the Creative Commons Attribution 4.0 International License (http://creativecommons.org/licenses/by/4.0/), which permits unrestricted use, distribution, and reproduction in any medium, provided you give appropriate credit to the original author(s) and the source, provide a link to the Creative Commons license, and indicate if changes were made. The Creative Commons Public Domain Dedication waiver (http://creativecommons.org/publicdomain/zero/1.0/) applies to the data made available in this article, unless otherwise stated. 


\section{Background}

Non-alcoholic steatohepatitis (NASH) is part of the spectrum of non-alcoholic fatty liver disease (NAFLD) [1]. Definitive diagnosis of NASH requires a liver biopsy and is established based the minimum criteria of $5 \%$ of tissue with fat (steatosis); presence of lobular inflammation; and hepatocellular injury termed "ballooning degeneration" [2]. When evaluating for this diagnosis, histologic assessment of steatosis and inflammatory cell quantification is relatively straight forward [3], leading to minimum of variation in scoring [4]. Ballooning degeneration, on the other hand can be subtle and difficult to detect and quantify leading to significant inter-observer inconsistency [5-7].

The difficulty in assessing ballooning degeneration arises from its variable presentation, combined with a largely descriptive definition that lack consensus regarding underlying pathogenesis [8]. Ballooned hepatocytes are typically large round cells with a reticulated cytoplasm on hematoxylin and eosin (HE)-stained sections [9]. Specifically, hepatocyte ballooning degeneration is characterized by visible swelling of the hepatocyte and vacuolization with clear cytoplasm. In some cells, CK18 intermediate filament loss accompanies ballooning [9]. However, the morphological features of ballooning degeneration can be mimicked by glycogenated hepatocytes or microvesicular fatty changes in hepatocytes [10].

NASH has been considered the progressive form of NAFLD $[1,2]$. In this context, most of the therapeutic clinical trials have focused on identifying patients with NASH [11]. On the other hand, severity of hepatic fibrosis has been shown to determine the long-term outcome
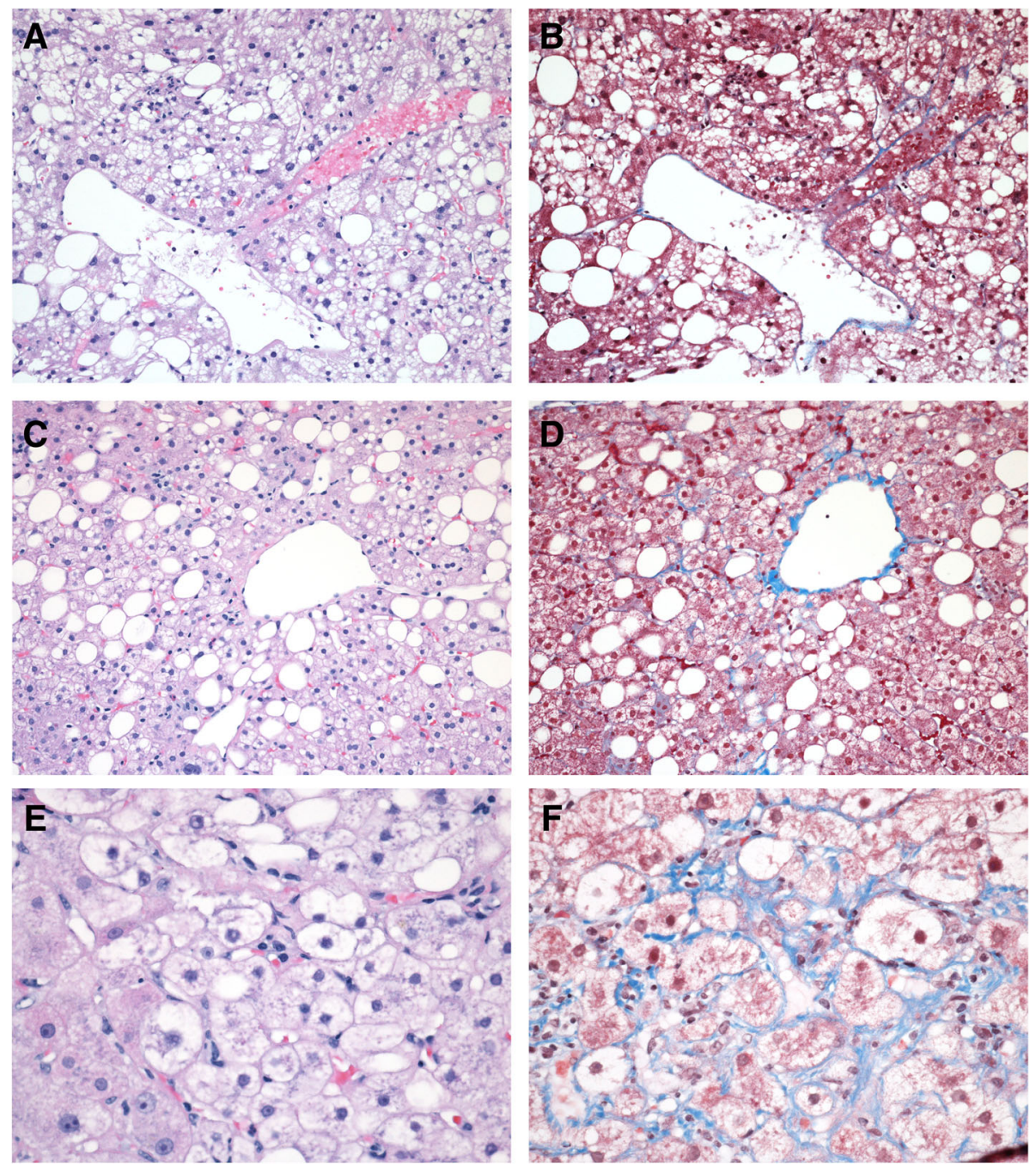

Fig. 1 Representative Masson trichrome and H\&E stained liver biopsies of NALFD, "borderline" NASH, and NASH: a, c, and e images are h\&e stains, while $\mathbf{b}$, d, and $\mathbf{f}$ are the correlate Masson trichrome. $\mathbf{a}$ and $\mathbf{b}$ (both 20X) are of a Simple steatosis or non-NASH NAFLD patient. $\mathbf{c}$ and $\mathbf{d}$ (both 20X) are from a "borderline" NASH patient having steatosis and pericellular fibrosis but no ballooning degeneration. $\mathbf{e}$ and $\mathbf{f}(40 \mathrm{X})$ are from a NASH patient and show ballooning degeneration, pericellular fibrosis, and steatosis 
of NAFLD [4, 12-14]. Nevertheless, because ballooning degeneration is independently associated with hepatic fibrosis [15], it seems likely that the type of hepatocellular injury that results in ballooning may simultaneously stimulate fibrogenesis. Consequently, a histologic stain that reliably identifies mild forms of ballooning that is typically difficult to identify with routine staining would be of great help to establish the diagnosis of NASH. Furthermore, quantification of the degree of staining for ballooning degeneration may be helpful for assessing the severity of NASH.

A possible candidate for identification and quantification of hepatocyte ballooning is detection of hepatocyte Sonic Hedgehog signaling protein $(\mathrm{SHH})$. In a study by Guy et al. [16], qualitative assessment of SHH signal by IHC in liver biopsies correlated with the diagnosis of NASH as well as response to therapy. In the current investigation immunostained hepatic SHH was quantified using computer assisted morphometry (CAM). The pilot study assessed whether $\mathrm{SHH}$ quantity indicates severity of the disease as determined by circulating cytokeratin 18 (M65), its caspase degradation product (M30) which have been shown to correlate with histologic severity in NAFLD $[17,18]$ as well as pathologic evaluations.

\section{Methods}

\section{Subjects}

Patients with biopsy proven NAFLD and control subjects with no or minimal histologic abnormalities were included in the study $(N=69)$. The biopsies were evaluated by single hepatopathologist. Fasting serum samples that had been collected within 3 months of the biopsy were available along with clinical and laboratory data. Biopsies were a mixture of needle core and wedge, with the needle core having an average length of $18.8 \mathrm{~mm}$. Exclusion criteria included inadequate biopsy, excessive use of alcohol, history of viral hepatitis or other liver diseases. All patients participating in this study were pre-consented in accordance with our institutional review board.

\section{Measurement of circulating Analytes}

Using fasting serum, circulating analytes [M30 Apoptosense $^{\circ}$ CK18 Kit (DiaPharma, OH) and M65 EpiDeath ${ }^{\circ}$ ELISA (DiaPharma, OH)] were measured by ELISA. Circulating $\mathrm{SHH}$ concentrations were measured by ab100639 - Sonic Hedgehog Human ELISA Kit (Abcam, MA). All protocols were performed as per manufacturers' instructions.

\section{Immunohistochemistry and histologic assessment}

Histologic assessment included semiquantitative scoring of the following parameters: steatosis (estimated proportion of parenchyma occupied by fat) on a scale of $0-4$ with
$0=$ none, $1=>0$ but $<5 \%, 2=5-33 \%, 3=34-66 \%, 4=>$ $66 \%$; lobular and portal inflammation, and features of hepatocellular injury (apoptosis, ballooning, Mallory-Denk bodies) on a scale of $0-3$ with $0=$ none, $1=$ mild or few, 2 $=$ moderate, $3=$ marked or many. Components of fibrosis were graded separately with centrilobular pericellular/ perisinusoidal fibrosis typical of early stage NAFLD and portal fibrosis scored from 0 to 3 for none, mild, moderate and marked. Pre-cirrhotic bridging fibrosis was scored as 0 (absent), 1 (few = bridges linking fewer than $50 \%$ of central veins and portal tracts), or 2 (many=linkage of greater than $50 \%$ of central veins and portal tracts. Cirrhosis was scored as 0 (absent), 1 (early or incomplete) or 2 (established or advanced). Most cirrhotic liver biopsies also had portal fibrosis scores of 3 , since the portal tracts were typically incorporated into the cirrhotic septa.

Immunohistochemical staining for $\mathrm{SHH}$ was done by standard procedure using anti-SHH antibody (Abcam, MA; ab53281) at a dilution of 1:4000. Briefly, following deparaffinization and rehydration, epitope retrieval was performed in a steamer by incubating slides in IHC-TEK Epitope retrieval solution (IHCWorld, MD) for $40 \mathrm{~min}$. After rinsing, slides were blocked using $3 \% \mathrm{H}_{2} \mathrm{O}_{2}$ for 30 min, then rinsed again. Primary antibody was applied and slides were allowed to incubate for $60 \mathrm{~min}$ in a humidified chamber. Following successive wash steps, secondary antibody was applied and allowed to incubate for 30 min (EnVision ${ }^{\mathrm{nt}}+$ Dual Link System-HRP, Dako, CA). ImmPACT DAB (Vector Laboratories, CA) was used for detection, and slides were counterstained with hematoxylin before dehydration and mounting.

Post-staining, a digitized image of the entire section was acquired using an Aperio/Leica Scanscope XT scanner at 40X magnification. Twelve annotations of equal

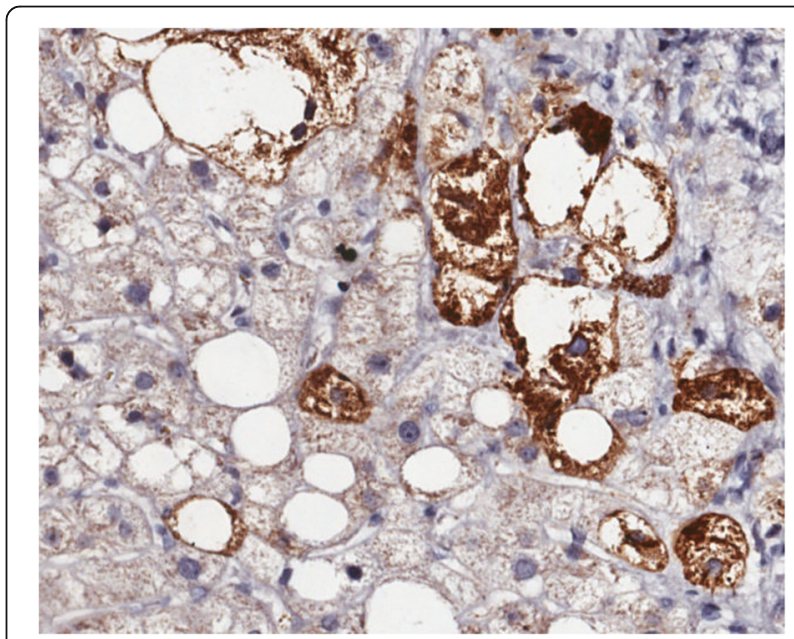

Fig. 2 3,3'-Diaminobenzidine (DAB) staining of liver tissue using the antibody ab53281 (Brown). Staining is specific to hepatocytes undergoing ballooning degeneration 
Table 1 Correlations between SHH or pathology scores for pericellular fibrosis, ballooning degeneration and Circulating Analytes

\begin{tabular}{|c|c|c|c|c|c|c|}
\hline \multirow{2}{*}{$\begin{array}{l}\text { Circulating } \\
\text { Factor }\end{array}$} & \multicolumn{2}{|l|}{$\mathrm{SHH}$} & \multicolumn{2}{|c|}{ Ballooning Degeneration } & \multicolumn{2}{|c|}{ Pericellular Fibrosis } \\
\hline & rho & $P$-Value & rho & $P$-Value & rho & $P$-Value \\
\hline M65 & 0.588 & $P<0.0001$ & 0.538 & $P<0.0001$ & 0.548 & $P<0.0001$ \\
\hline M30 & 0.375 & $P=0.001$ & 0.483 & $P<0.0001$ & 0.525 & $P<0.0001$ \\
\hline AST & 0.43 & $P<0.0004$ & 0.41 & $P<0.0004$ & 0.50 & $P<0.0001$ \\
\hline ALT & 0.27 & $P<0.03$ & 0.18 & $P=0.07$ & 0.31 & $P=0.01$ \\
\hline
\end{tabular}

area consisting of typical hepatic parenchyma were chosen at random and distributed throughout the biopsy; placement of these areas of quantification was done by two separate researchers working independently to assess methodological robustness. An area quantification algorithm was then used to quantify the degree of $\mathrm{SHH}$ expression in these annotated areas.

\section{Statistical analysis}

Clinico-demographic and laboratory parameters were compared between subjects with and without NASH using chi-square test (for categorical parameters) or Mann-Whitney test (for continuous parameters). Correlations between continuous parameters were calculated using Spearman's non-parametric approach. Independent predictors of NASH and advanced fibrosis in patients with NAFLD were assessed using multiple logistic regression models with bidirectional stepwise selection of parameters; only those with $p<0.05$ were left in the models. All analyses were run in SAS 9.3 (SAS Institute, Cary, NC).

\section{Results}

\section{Cohort characteristics}

Clinical and demographic data for the study subjects are as follows: Caucasian $=79.4 \%$, female $=66.1 \%$, age $=46 \pm$ 11 years, $\quad$ ALT $=50.2 \pm 43.3 \mathrm{U} / \mathrm{L}, \quad$ AST $=38.8 \pm 33.9 \mathrm{U} / \mathrm{L}$ and fasting serum glucose $=110.2 \pm 39.6 \mathrm{mg} / \mathrm{dL}$. Of the study group, 19 subjects had minimal histologic changes that did not meet the criteria for a pathologic diagnosis (i.e. no discernable liver disease), while 50 (73.5\%) had NAFLD. Among patients that had NAFLD, 44 (64.7\%) had histologic NASH. According to the NASH CRN criteria, 16 patients $(23.5 \%)$ were considered to have borderline NASH while 28 (41.2\%) had steatosis, lobular

Table 2 Correlations between $\mathrm{SHH}$ and Histologic Lesions

\begin{tabular}{lll}
\hline Hepatic Lesion & rho & $P$-Value \\
\hline Ballooning Degeneration & 0.62 & $P<0.0001$ \\
Steatosis & 0.554 & $P<0.001$ \\
Mallory-Denk bodies & 0.54 & $P<0.001$ \\
Pericellular fibrosis & 0.527 & $P<0.001$ \\
Lymphocytic infiltration & 0.435 & $P<0.002$ \\
\hline
\end{tabular}

inflammation and unequivocal hepatocellular ballooning and would be considered definite NASH (Fig. 1).

As expected, circulating M30 and M65 were significantly higher in NASH patients as compared to those with non-NASH. In fact, serum levels of M30 were almost twice as high in NASH patients $(539.1 \pm 290.8 \mathrm{U} / \mathrm{L}$ vs. $287.6 \pm 190.5 \mathrm{U} / \mathrm{L} ; p=0.0002)$, M65 was close to three times higher in NASH patients compared to all non-NASH subjects $(441.2 \pm 464.2 \mathrm{U} / \mathrm{L}$ vs. $162.8 \pm 353.1$ $\mathrm{U} / \mathrm{L} ; p=0.0006)$. In contrast, circulating $\mathrm{SHH}$ failed to show differential concentrations between $\mathrm{NASH}$ and non-NASH subjects.

\section{Assessment of hepatic SHH}

We also performed and assessed immunostaining for hepatic $\mathrm{SHH}$ on the liver biopsies. For further assessment of SHH signal, only slides from NAFLD subjects were considered partially because of the potential for bias caused by including 19 no-disease subjects whose biopsies lacked positive signal for $\mathrm{SHH}$, and partially because examining $\mathrm{SHH}$ signal gradient among the NAFLD was of greater research interest. In this context, staining for $\mathrm{SHH}$ with ab53281 produced intense positive signal in the cytoplasm of a subset of hepatocytes undergoing ballooning degeneration, and was not seen to bind directly with Mallory-Denk bodies (Fig. 2). Additionally, a weak but consistent signal could be detected in bile duct epithelial cells, which served as a useful technical internal control. Hepatic $\mathrm{SHH}$ expression ranged from 0 to $8.4 \%$ of the area scanned with an average of $0.97 \% \pm 1.8$. Inter-observer variation between observers was very low ( $\mathrm{rho}=0.939$, $P<0.0001)$.

Interestingly, degree of hepatic $\mathrm{SHH}$ signal as detected by CAM correlated with several circulating biomarkers associated with apoptosis: hepatic $\mathrm{SHH}$ signal positively correlated with circulating M65 ( $\mathrm{rho}=0.588, \mathrm{P}<0.0001$ ), as well as circulating M30 (rho $=0.375, P=0.001$ ). Hepatic SHH signal also modestly correlated with circulating AST and ALT (rho $=0.43, P=0.0004$, and rho $=0.27, P=0.03$, respectively) (Table 1). Hepatic $\mathrm{SHH}$ signal did not significantly correlate with circulating $\mathrm{SHH}$ levels as measured by ELISA. Interestingly, the pathologist assessment of ballooning degeneration showed significant correlation to only circulating M65, M30, and AST (rho = 0.52, $P<0.0001$; rho = 

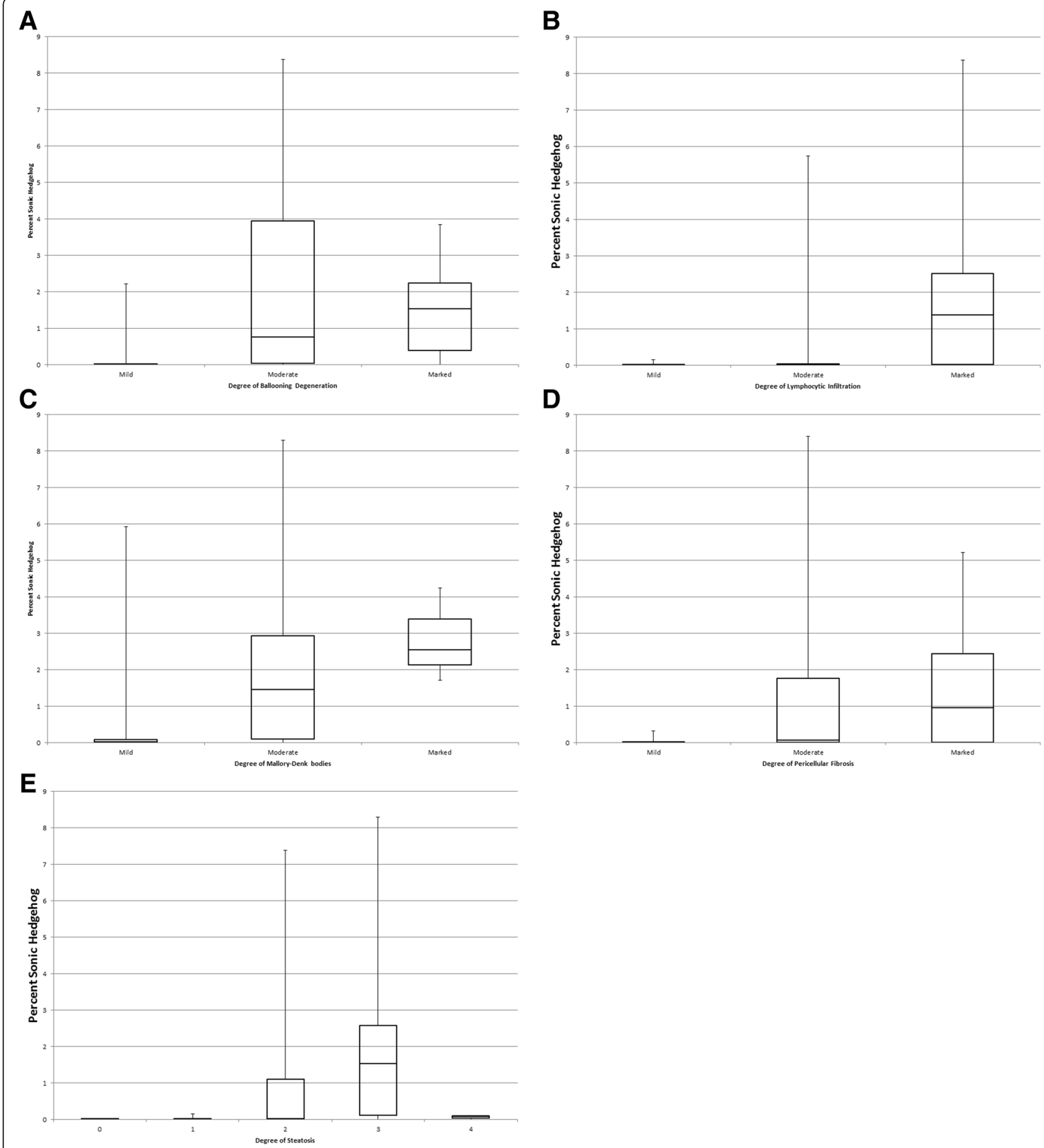

Fig. 3 Box and Whisker plots of percent SHH vs. pathologist's assessment of hepatic lesions: a Ballooning degeneration, b Lymphocytic infiltration, c MalloryDenk bodies, $\mathbf{d}$ Pericellular fibrosis, e Hepatic steatosis

0.48, $P<0.0001$; rho $=0.41, P=0.0005$, respectively). Unlike hepatic $\mathrm{SHH}$ signal, no correlation was detected between histopathological ballooning assessment and ALT levels.

Hepatic SHH signal also correlated with a number of liver injury markers. Hepatic SHH signal was strongly correlated to histologic ballooning degeneration $(\mathrm{rho}=0.62$, $p<0.0001)$. Additionally, a modest but significant correlation was seen with the grade of steatosis (rho $=0.554, p<$ 0.001 ), Mallory-Denk bodies ( $\mathrm{rho}=0.54, \mathrm{p}<0.001$ ), pericellular fibrosis (rho $=0.527, \mathrm{p}<0.001$ ), and lymphocytic infiltration (rho $=0.435, p<0.0002)$ (Table 2, Fig. 3). Although hepatic $\mathrm{SHH}$ signal did correlate with 
Table 3 Distribution of Patients by SHH tertile or assessment of ballooning degeneration and degree of pericullar fibrosis

\begin{tabular}{|c|c|c|c|c|c|c|}
\hline \multirow[t]{2}{*}{ Degree of Pericellular Fibrosis } & \multicolumn{3}{|c|}{ SHH Tertile } & \multicolumn{3}{|c|}{ Degree of Ballooning Degeneration } \\
\hline & $1 \mathrm{st}$ & 2nd & $3 r d$ & Mild & Medium & Marked \\
\hline Mild & 16 & 9 & 0 & 24 & 0 & 1 \\
\hline Medium & 5 & 8 & 7 & 8 & 10 & 2 \\
\hline Marked & 4 & 4 & 12 & 6 & 9 & 5 \\
\hline
\end{tabular}

Mallory-Denk bodies, histologically determined ballooning degeneration much more strongly correlated with Mallory-Denk bodies (rho $=0.73, p<0.0001$ ).

Although hepatic $\mathrm{SHH}$ immunostaining and histologic assessment of ballooning degeneration similarly correlated with the histologic assessment of hepatic pericellular fibrosis, the relationship may be more nuanced (Table 3); Degree of pericellular fibrosis among subjects based on their hepatic SHH signal and histopathological assessment of ballooning degeneration was evaluated (Table 3). Higher number of patients with mild/undetectable ballooning degeneration had mild/undetectable pericellular fibrosis $(N=24)$, when compared to those with the lowest $\mathrm{SHH}$ tertile $(N=16)$. However, larger number of patients had marked pericellular fibrosis in the highest $\mathrm{SHH}$ tertile $(N=12)$ when compared to histopathological assessment of marked ballooning degeneration $(N=5)$. Further, logistic modeling indicated hepatic SHH signal and presence of diabetes were both independent predictors of advanced fibrosis (portal fibrosis $>2$ and pericellular fibrosis scores $>2$ : OR $=1.986$, $p=0.01$, and $\mathrm{OR}=3.280, p=0.03$, respectively).

\section{Discussion}

The application of computer assisted image analysis with morphometric tools can provide accurate measurements of pathologic features of liver biopsy on a continuous scale, as opposed to a categorical one, which will allow broader statistical analysis and comparisons between studies. The intent of this pilot study was to assess if the benefits of computer-assisted digital image analysis could be realized in the measurement of ballooning degeneration, a key pathologic component of $\mathrm{NASH}$, by quantification of $\mathrm{SHH}$ detection.

Our data shows significant correlation between the degrees of hepatic SHH signal and histopathologic lesions (ballooning degeneration and fibrosis). Correlations were also seen between CAM measured hepatic SHH signal and circulating forms of the epithelial cell structural protein cytokeratin18 associated with the progressive NAFLD (M30 and M65). These data warrant further validation of CAM measured hepatic SHH signal as a tool to more accurately diagnose and grade NASH. The close association between pericellular or advanced fibrosis and hepatic $\mathrm{SHH}$ quantification by CAM may lead to a more useful measure to parse patient populations regarding clinically important outcomes.

Though measurement of circulating cytokeratin 18 by M65 and its caspase degradation product by M30 are correlated with $\mathrm{SHH}$ staining, approximately half of the patients with had very low or negative SHH by CAM, suggesting that ballooning is not a direct source of these biomarkers and indicating that blood levels do not necessarily indicate marked histologic ballooning.

Although this research was not intended to explore the role of $\mathrm{SHH}$ in the pathogenesis of $\mathrm{NASH}$, our study may offer some insight for future studies. The antibody used to detect $\mathrm{SHH}$ in this study (ab53281) binds in the $\mathrm{C}$-terminus region of the Pro-SHH peptide, detecting either the full length pro-SHH peptide or the $27 \mathrm{kDa}$ C-terminal fragment $\left(\mathrm{SHH}_{\mathrm{c}}\right)$. The measurable accumulation of either of these products within a subset of hepatocytes undergoing ballooning degeneration is interesting, especially given that attempts to detect the cleaved $19 \mathrm{kDa}$-terminal fragment (using SC-9024) on serial sections were unsuccessful (Data not shown). Notably, no correlations were found between circulating serum $\mathrm{SHH}$ and hepatic $\mathrm{SHH}$ signal. This suggests the possibility of derangements in $\mathrm{SHH}$ post-translational modification in ballooning hepatocytes, perhaps in cholesterol addition / cleavage process of full length $\mathrm{SHH}$ into $\mathrm{N}$ and $\mathrm{C}$ fragments, or the proteasome degradation of $\mathrm{SHH}_{\mathrm{c}}$. If so, the accumulation of $\mathrm{SHH}$ or $\mathrm{SHH}_{\mathrm{c}}$ within ballooning hepatocytes could theoretically be a marker for those NAFLD patients in danger of progressive fibrosis.

Our study does have a few limitations. Although the sample size relatively small, we believe that the results of this pilot study can inform larger studies to validate the role of $\mathrm{SHH}$ staining in $\mathrm{NASH}$. In addition, evaluation of any hypotheses regarding the role of $\mathrm{SHH}$ in the pathogenesis of NASH or fibrosis, or of its predictive value will require further experimentation over multiple time points and stages of disease.

\section{Conclusion}

In summary, computer assisted quantitation of hepatic $\mathrm{SHH}$ correlates with liver lesions and circulating analytes associated with NASH. Although a larger patient population with biopsy specimens from multiple times will be needed to truly assess $\mathrm{SHH}$ as a grading and staging tool 
for NALFD, our findings are a step toward the ultimate goals of increasing the resolution of the liver biopsy reading and eliminating inter- and intra-observer variability as it pertains to ballooning assessment and NAFLD diagnosis.

\section{Abbreviations}

ALT: Alanine Aminotransferase; AST: Aspartate Aminotransferase; CAM: Computer assisted morphometry; CK18: Cytokeratin 18; DAB: 3,3'Diaminobenzidine; ELISA: Enzyme-linked Immunosorbent Assay; IHC: Immunohistochemistry; NAFLD: Non-alcoholic fatty liver disease; NASH CRN : Clinical Research Network in Nonalcoholic Steatohepatitis; NASH: Nonalcoholic steatohepatitis; SHH: Sonic Hedgehog; SHHc: Sonic Hedgehog Cterminal fragment

\section{Acknowledgements}

Not applicable.

\section{Availability of data and material}

All data generated or analysed during this study are included in this published article.

\section{Funding}

This study has been supported by the Beatty Liver and Obesity Fund at Inova Health System and the Liver Disease Outcomes Fund of the Center for Liver Diseases at Inova Fairfax Hospital, Falls Church, VA. The funding body did not participate in the design or implementation of this study.

\section{Authors' contributions}

ME and RM conceived the study, performed immunostaining, participated in study design, coordinated all efforts, drafted and edited the manuscript. GB optimized and assisted in immunostaining. FM, SA, DA, assisted in biopsy slide storage and labeling during computer assisted morphometry.LA performed computer assisted morphometry. ZY collected informed consent and performed sample processing. MS performed statistical analysis. ZG read the liver biopsies. ZG and ZYM participated in the conception and design of the study and finalized the manuscript. All authors read and approved the final manuscript.

\section{Ethics approval and consent to participate}

This study has been approved by Internal Review Board of Inova Fairfax Hospital (Federal Assurance FWA00000573). Sample collection and storage was approved by Inova IRB \#05.047. Written informed consent for sample collection and use of samples for research at the Betty and Guy Beatty Center for Integrated Research, Inova Fairfax Medical Campus facility was obtained from all patients included in this study. The samples were deidentified in compliance with HIPAA regulations.

\section{Consent for publication}

Not applicable.

\section{Competing interests}

The authors declare that they have no competing interests.

\section{Publisher's Note}

Springer Nature remains neutral with regard to jurisdictional claims in published maps and institutional affiliations.

\section{Author details}

'Center for Liver Diseases, Department of Medicine, Inova Fairfax Hospital, Virginia, USA. ${ }^{2}$ Betty and Guy Beatty Center for Integrated Research, Claude Moore Health Education and Research Building, Inova Health System, 3300 Gallows Road, Falls Church, VA 22042, USA. ${ }^{3}$ Center for Outcomes Research Liver Diseases, Washington, DC, USA.
Received: 21 September 2018 Accepted: 1 February 2019 Published online: 11 February 2019

\section{References}

1. Chalasani N, Younossi Z, Lavine JE, Charlton M, Cusi K, Rinella M, et al. The diagnosis and management of nonalcoholic fatty liver disease: Practice guidance from the American Association for the Study of Liver Diseases. Hepatology [Internet]. 20181 [cited 2018 Feb 7];67(1):328-57. Available from: https:/doi.org/ 10.1002/hep.29367/abstract.

2. Younossi ZM, Loomba R, Anstee QM, Rinella ME, Bugianesi E, Marchesini G, et al. Diagnostic modalities for non-alcoholic fatty liver disease (NAFLD), non-alcoholic steatohepatitis (NASH) and associated fibrosis. Hepatol Baltim Md. 2017.

3. Caldwell S, Lackner C. Perspectives on NASH histology: cellular ballooning. Ann Hepatol. 2017 Apr;16(2):182-4.

4. Younossi ZM, Stepanova M, Rafiq N, Makhlouf H, Younoszai Z, Agrawal R, et al. Pathologic criteria for nonalcoholic steatohepatitis: Interprotocol agreement and ability to predict liver-related mortality. Hepatology [Internet]. 2011 [cited 2014 13];53(6):1874-1882. Available from: https://doi.org/10.1002/hep.24268/abstract.

5. Jung ES, Lee K, Yu E, Kang YK, Cho M-Y, Kim JM, et al. Interobserver Agreement on Pathologic Features of Liver Biopsy Tissue in Patients with Nonalcoholic Fatty Liver Disease. J Pathol Transl Med [Internet]. 2016 May [cited 2017 Dec 13]:50(3):190-6. Available from: https://www.ncbi.nlm.nih. gov/pmc/articles/PMC4876083/.

6. Younossi ZM, Gramlich T, Liu YC, Matteoni C, Petrelli M, Goldblum J, et al. Nonalcoholic fatty liver disease: assessment of variability in pathologic interpretations. Mod Pathol Off J U S Can Acad Pathol Inc. 1998;11(6):560-5.

7. Fukusato T, Fukushima J, Shiga J, Takahashi Y, Nakano T, Maeyama S, et al. Interobserver variation in the histopathological assessment of nonalcoholic steatohepatitis. Hepatol Res Off J Jpn Soc Hepatol. 2005;33(2):122-7.

8. Caldwell S, lkura Y, Dias D, Isomoto K, Yabu A, Moskaluk C, et al. Hepatocellular ballooning in NASH. J Hepatol. 2010 Oct:53(4):719-23.

9. Lackner C, Gogg-Kamerer M, Zatloukal K, Stumptner C, Brunt EM, Denk H. Ballooned hepatocytes in steatohepatitis: the value of keratin immunohistochemistry for diagnosis. J Hepatol. 2008 May;48(5):821-8.

10. Yeh MM, Brunt EM. Pathology of Nonalcoholic Fatty Liver Disease. Am J Clin Pathol [Internet]. 20071 [cited 2017 Dec 13];128(5):837-47. Available from: https://academic.oup.com/ajcp/article/128/5/837/1760386.

11. Younossi ZM, Loomba R, Rinella ME, Bugianesi E, Marchesini G, Neuschwander-Tetri BA, et al. Current and future therapeutic regimens for non-alcoholic fatty liver disease (NAFLD) and non-alcoholic steatohepatitis (NASH). Hepatol Baltim Md. 2017 Dec;9.

12. Angulo P, Kleiner DE, Dam-Larsen S, Adams LA, Bjornsson ES, Charatcharoenwitthaya P, et al. Liver Fibrosis, but No Other Histologic Features, Is Associated With Long-term Outcomes of Patients With Nonalcoholic Fatty Liver Disease. Gastroenterology. 2015 149(2):389-397.e10

13. Ekstedt M, Hagström H, Nasr P, Fredrikson M, Stål P, Kechagias S, et al. Fibrosis stage is the strongest predictor for disease-specific mortality in NAFLD after up to 33 years of follow-up. Hepatol Baltim Md. 2015;61(5): 1547-54.

14. Younossi ZM, Stepanova M, Rafiq N, Henry L, Loomba R, Makhlouf H, et al. Nonalcoholic steatofibrosis independently predicts mortality in nonalcoholic fatty liver disease. Hepatol Commun [Internet]. 20171 [cited 2017 Dec 13]; 1(5):421-8. Available from: https://doi.org/10.1002/hep4.1054/abstract.

15. Gramlich T, Kleiner DE, McCullough AJ, Matteoni CA, Boparai N, Younossi ZM. Pathologic features associated with fibrosis in nonalcoholic fatty liver disease. Hum Pathol [Internet]. 20041 [cited 2017 Dec 13];35(2):196-9. Available from: http://www.humanpathol.com/article/S0046-8177(03)006099/fulltext.

16. Guy CD, Suzuki A, Zdanowicz M, Abdelmalek MF, Burchette J, Unalp A, et al. Hedgehog pathway activation parallels histologic severity of injury and fibrosis in human nonalcoholic fatty liver disease. Hepatol Baltim Md. 2012; 55(6):1711-21.

17. Wieckowska A, Zein NN, Yerian LM, Lopez AR, McCullough AJ, Feldstein AE. In vivo assessment of liver cell apoptosis as a novel biomarker of disease severity in nonalcoholic fatty liver disease. Hepatol Baltim Md. 2006;44(1): 27-33.

18. Shen J, Chan HL-Y, Wong GL-H, Chan AW-H, Choi PC-L, Chan H-Y, et al. Assessment of non-alcoholic fatty liver disease using serum total cell death and apoptosis markers. Aliment Pharmacol Ther [Internet]. 20121 [cited 2018 Feb 9];36(11-12):1057-66. Available from: https:/doi.org/10.1111/apt. 12091/abstract. 